\title{
DETAILS OF INVESTIGATION
}

\section{a) Objectives}

To identify constituents of human olfactory mucosal cultures, characterise the behaviour of human cultured OECs (hOECs) in vitro assays, establish GMP production of hOECs, and perform "first-in-man" studies of hOEC transplantation for brachial plexus repair.

\section{b) Milestones}

1 (24 months) Characterisation of cell types in cultured olfactory mucosa, using confocal microscopy, flow cytometry and microbioreactor techniques.

(24 months) GMP paperwork ready for submission to HTA and MHRA for registration.

2 (36 months) Functional testing of olfactory mucosal cultures, assessing the effect of different hOEC:fibroblast ratios on neurite outgrowth in spinal cord slice culture models.

(36 months) Registration of GMP cleanroom with MHRA/HTA

3 (48 months) Completion of work packages

\section{c) Method}

\section{Work package 1}

Characterisation of cells obtained from cultures of human olfactory mucosa Human specimens $\mathrm{n}=20$

The olfactory mucosa contains many different cell types [Chen 2014, Holbrook 2011] (figure 1).

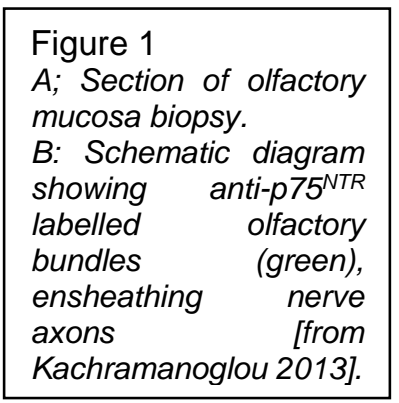
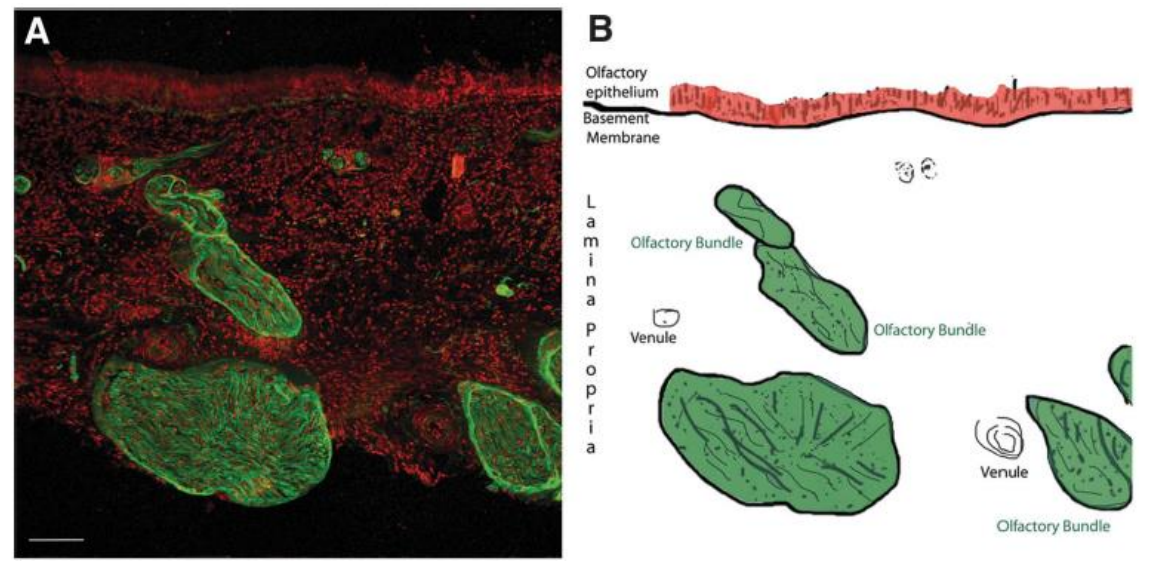

We will harvest olfactory mucosa biopsies from patients who are undergoing endoscopic surgery of the nasal sinuses, skull base or pituitary gland region. Previous studies have revealed the majority of cells from mucosa comprise of hOECs and fibroblasts (figure 2) but there are a number of unidentified cells, similar to those found in rat OEC cultures [Kueh 2011] which we will identify in human samples by immunostaining. We obtain one patient sample every 1-2 weeks and will analyse 20 samples in 12 months to establish the expected yields and variation of different cells from the mucosa. 

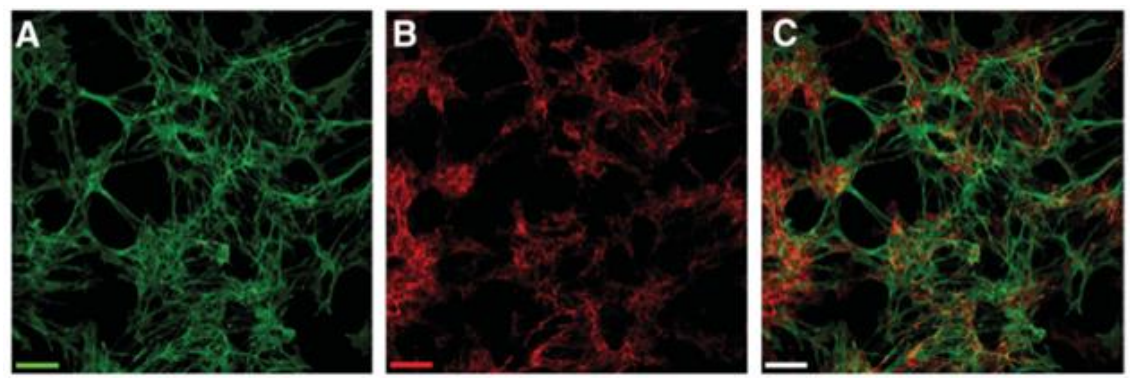

Figure 2

Human OEC cultures

A: green OECs, anti-

p75 labelling.

$B$ : red fibroblasts, antifibronectin.

C: superimposed

The human specimens will be bisected, and half will be cryosectioned for immunohistological labelling using a battery of antibody labels (below) to define the cellular composition of our starting material, imaged using a Zeis Axioscan slidescanner. Manual cell counts will be performed on 5 high-power fields for each $35 \mathrm{~mm}$ dish. Olfactory marker protein and S100 labelling will be quantified in a minimum of three tissue sections per sample to evaluate whether the proportion of these antigens in biopsy sections can be used to predict the proportion of hOECs in resulting cultures, as a potential rapid screening tool for the clinic.

The other half will be cultured by our standard technique [Choi 2008] and cultured cells will be separated into alliquots for testing two antibodies at a time, analysed by confocal microscopy. During the initial phases of our standard culture technique, two differential adhesion steps are used to remove cells that quickly adhere to the culture dish within the first 18-36 hours. Since OECs take several days to adhere, this enriches the culture for OECs, but we will also analyse the discarded cells.

We will assess the expected proportions of different cell types in the final mixed cell culture, and whether there is any correlation between the initial histological assessment, waste cells (from the differential adhesions steps) and the final product.

Cell types will be identified with the following antibodies, to quantify the cell yields and allow us to determine product release criteria:

OECs (S100, GFAP, Sox10, O4, P75NTR, Vimentin antibodies)

Fibroblasts (CD90, Fibronectin, Vimentin)

Epithelial cells ( $\beta$-IV tubulin)

Endothelial cells (CD146)

Olfactory sensory neurons (OMP)

Glandular, sustentacular, and horizontal basal cells (Wide spectrum cytokeratin)

Horizontal basal cells (p63)

Globose basal cells (Mash1)

Schwann cells (HNK1)

\section{Work package 2}

Quantitative assessment of Human olfactory mucosa cultures Human specimens $\mathrm{n}=20$

For patient numbers 21-40, the mucosal samples will be dissociated and separated into two aliquots when there is sufficient material.

One aliquot will be analysed by flow cytometry techniques, and the second aliquot will be analysed by seeding onto microbioreactor devices. 
Flow cytometry will be performed, using antibodies which have been successfully used and titrated in work package 1, to identify and quantify specific cell types within the mixed cell cultures obtained from human olfactory mucosa. Specifically, a panel of markers will be used that can detect different subsets of cells that express various combinations of marker proteins. This will tell us whether there are different "types" of OECs and whether there are any coexpressing "fibroblast" markers that may indicate a stem cell phenotype. We will use 4 colour flow cytometry (BD Accuri C6) and, where necessary, more sophisticated multi-parameter 8 colour analysis (BD FACS Canto).

\section{Microbioreactor screening tool}

We will employ a microbioreactor that was developed with Prof Justin Cooper-White at University of Queensland, Australia (based on his patented technology developed for optimising cell culture media for stem cell differentiation). The device can screen multiple input conditions (such as media composition and cell culture substrate) and has already been validated using human mesenchymal stem cells cultured on microcarriers in different flow rates (figure 3) and on different microcarrier surfaces (figure 4). It is possible to characterise cell products in situ to determine optimal input conditions for downstream marker expression, or to identify specific cell types in the device by perfusing specific antibodies for identification. The device will support development of a robust OEC product in this project by enabling process development using small $(150 \mu \mathrm{L})$ quantities of precious material per device.
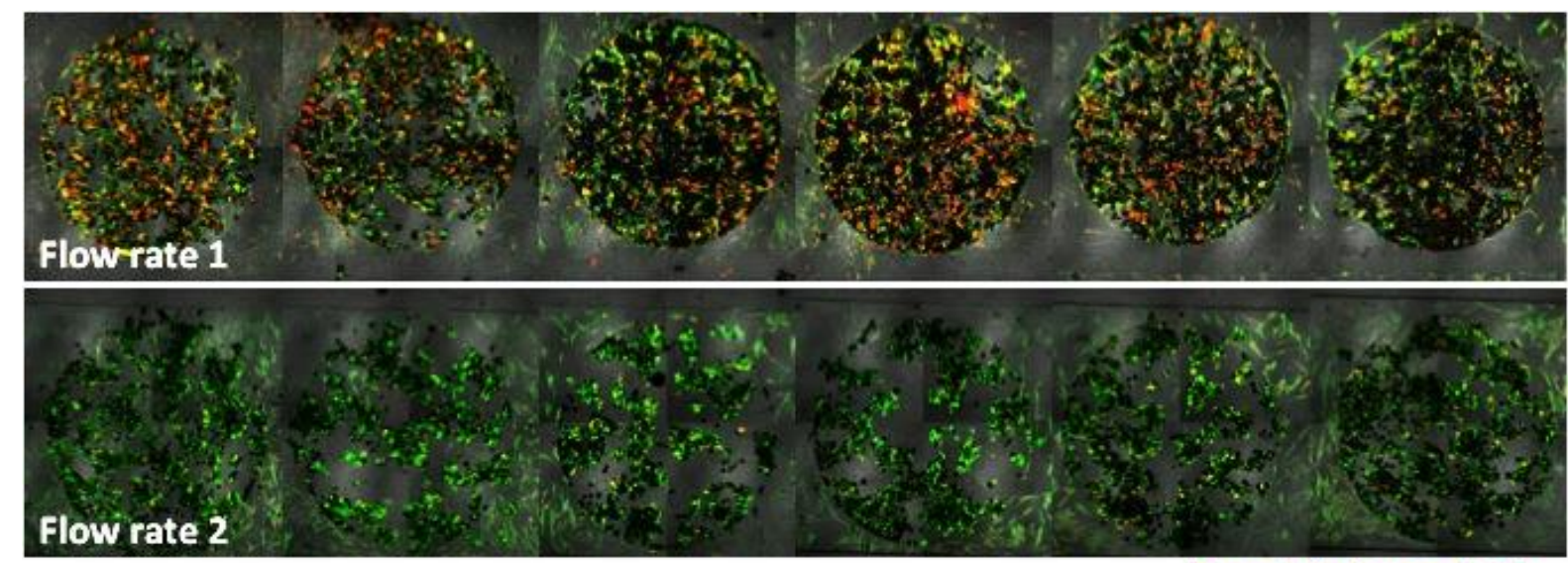

Type I Collagen Actin

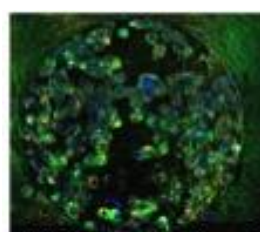

Microcarrier 1

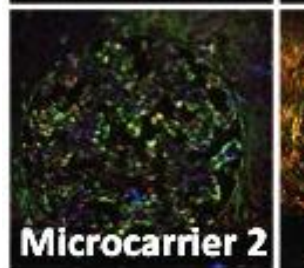

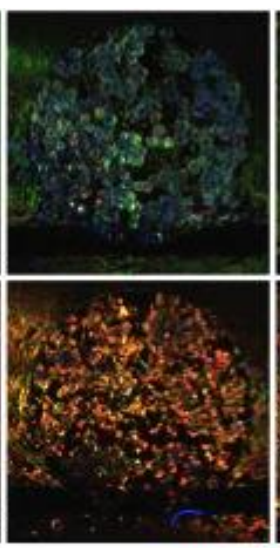

Type I Collagen Actin

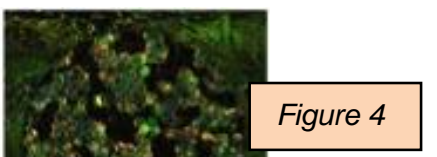

As well as cell identification, we can systematically test interactions between different media compositions (eg. commercial serum-free medias against serum-containing controls), media perfusion rates and cell culture substrates (extracellular matrix ligands, synthetic polymers and microcarriers) onto which the cells will adhere. 
Inlet

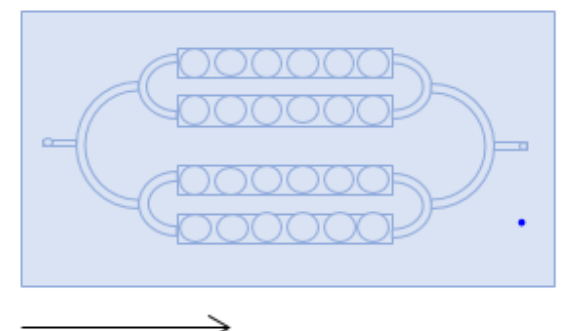

Direction of flow

\section{Outlet}

The following antibodies will be screened using the microbioreactor device, if the antibodies have been found to label human cells effectively, in dilutions determined in WP1:

P75, S100, GFAP, Sox10, O4

CD90, Thy1, Fibronectin, Vimentin

$\beta$-IV tubulin

Pancytokeratin

P53, Mash1, HNK1

\section{Work package 3}

In vitro studies - testing cells in a spinal cord slice culture model

We have developed an organotypic spinal cord slice culture assay to test the regenerative potential of the hOEC cell mixtures. We will assess the ability of hOEC cultures to promote motoneuron neurite outgrowth and to assess the impact of variations in hOEC:fibroblast proportions which will naturally vary between patients. We will assess the minimum proportion of hOECs required to produce an effect in this in vitro model. These experiments will provide validation data for future use of this assay system as a potency assay.

Initial experiments using this model indicated that rat OECs can enhance neurite outgrowth (figure 5) and that the model is capable of producing a dose response to known survival promoting factors (GDNF).

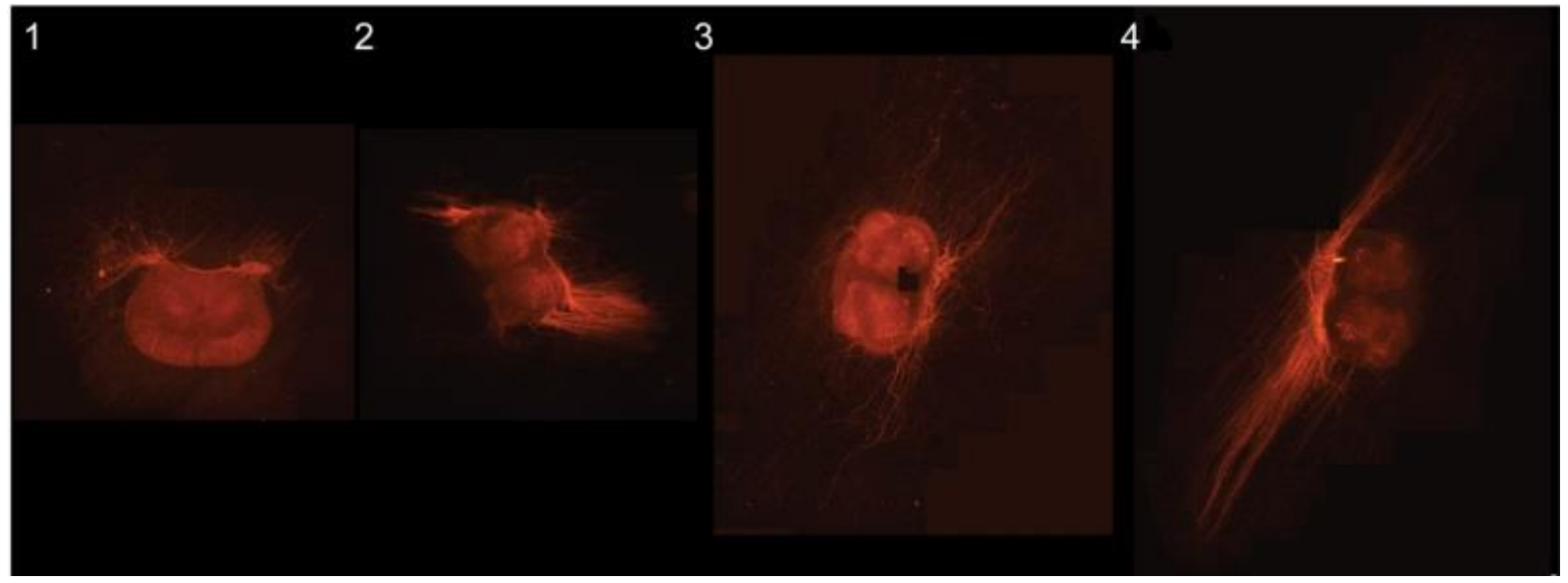

Figure 5

Slice cultures, stained with neurofilament antibodies, showing neurite outgrowth. 1- Collagen gel around the slice. 2- containing GDNF. 3- containing OECs. 4- containing both GDNF and OECs, showing further outgrowth. 
hOECs or control cells (3T3 fibroblasts) will be cultured on PLL coated cell culture membrane inserts. After 14 days, the co-cultures are fixed and analysed by immunofluorescence labelling. Neurites and ventral horn motoneurons are analysed by SMI32 and ChAT antibodies respectively. Each slice is imaged using NeuriteJ Scholl analysis to derive the maximum number of neurites, the maximal distance of growth, the Scholl critical value (distance where intersect the maximum number of neurites) and the Scholl regression coefficient (Change in neurite density with distance from explant) [Torres-Espín 2014]. The proportion of hOECs in the spinal cord slice co-cultures will be quantified by counting antihuman $\mathrm{p} 75^{\mathrm{NTR}}$ positive cells as a proportion of CM-Dil labelled cells in 5 random fields of view. 20 human biopsies will require $80-120$ slice cultures which would detect a $10 \%$ increase in neurite outgrowth $(\alpha=0.05, \beta=0.9)$.

\section{Work package 4}

GMP cell production

We have built a cleanroom at the National Hospital for Neurology and Neurosurgery that is designed for hOEC culture (figure 6), situated 2-3 minutes from the operating theatres. The room is presently undergoing operational qualification and performance qualification for MHRA and HTA licence application.

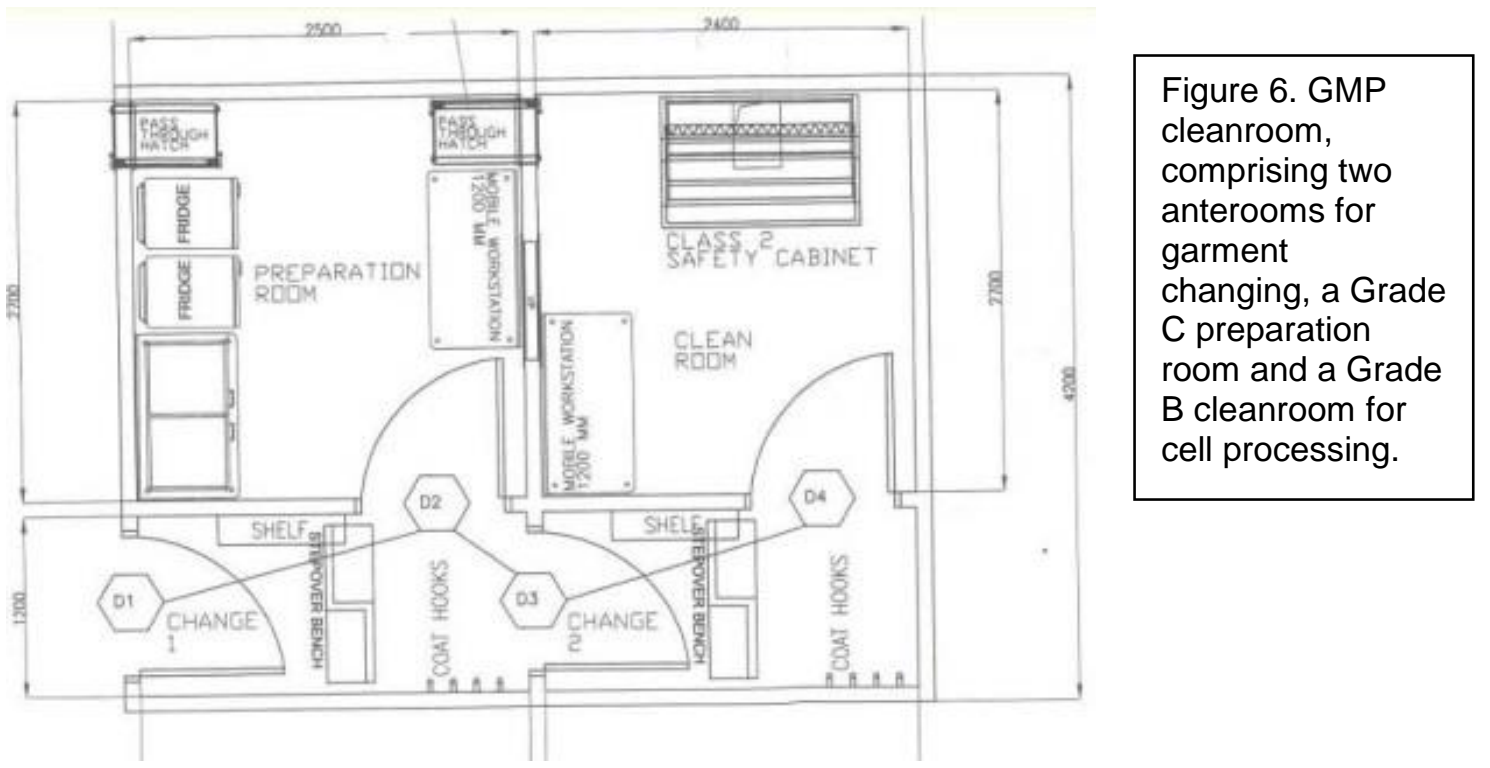

Quality assurance management systems have been designed, with rigorous standard operating procedures and production protocols. Particle counts and microbial monitoring are continuously performed. We will register and licence our cleanroom with the HTA and MHRA for a patient feasibility study and first-in-man testing of hOECs for brachial plexus injury.

Aspects of our quality management system which need to be finalised are:

- Validation of facility monitoring and cleaning standard operating procedures

- Performance qualification of the hOEC manufacturing process.

- Validation of protocols for safety testing and microbiological quality assessment of final cell product

- Collection of three months environmental monitoring for MHRA inspection. 


\section{Work Package 5}

Brachial Plexus re-implantation surgery with hOEC transplantation

Surgery to transplant hOECs in patients with traumatic brachial plexus avulsion will be performed on 2-3 consecutive patients to establish practicality and timing of biopsy, operation logistics (ad hoc theatre time, cell culture and product release, patient management) practicalities of outcome measures, and to advise on future clinical trial design. The reimplantation operation (without hOECs) has been performed on 27 patients at Queen Square as an NHS treatment, and the hOEC transplantation will be available as an NHS procedure, initially under an MHRA Specials licence.

Inclusion criteria: patients with brachial plexus avulsion. Exclusion criteria: age under 17 years, inability to give informed consent for any reason, pregnancy.

EQ-5D (Euroqol 5 dimensions) questionnaire will be used before and after surgery to document changes in overall quality of life. This will allow preliminary health economic analysis and calculation of cost per QALY gained, which is necessary to demonstrate prior to widespread NHS adoption. 